\title{
Molecular and Structural Characterization of Five Novel Mutations in the Bruton's Tyrosine Kinase Gene from Patients with X-Linked Agammaglobulinemia
}

\author{
Bratin K. Saha,* Sherill K. Curtis, ${ }^{*}$ Larry B. Vogler, ${ }^{\dagger}$ and \\ Mauno Vihinen ${ }^{*}$ \\ Departments of *Pathology and 'Pediatrics, Emory University, \\ Atlanta, Georgia, USA \\ ${ }^{\ddagger}$ Department of Biosciences, Division of Biochemistry, \\ University of Helsinki, Finland
}

\begin{abstract}
Background: The Btk (Bruton's tyrosine kinase) gene has been shown to be mutated in the human immunodeficiency disease, XIA (X-linked agammaglobulinemia). Btk is a member of the Tec family of cytosolic protein tyrosine kinases with distinct functional domains $\mathrm{PH}, \mathrm{TH}, \mathrm{SH} 3, \mathrm{SH} 2$, and kinase. Mutations have been observed in each of the Btk subdomains in XLA. We have analyzed the $B t k$ gene in six XLA patients from five unrelated families.

Materials and Methods: DNA was prepared from the patients' peripheral blood. The Btk exons including the junctional sequences were analyzed by single-strand conformation polymorphism (SSCP) followed by direct nucleotide sequencing after PCR-amplification. For structural analysis, the missense mutations were introduced into three-dimensional models of the PH and kinase domains of Btk and the outcome was predicted based on the knowledge of the protein function.

Results: Five novel mutations and two novel polymorphisms, all of which resulted from single-base alterations, were identified. Three of the five mutations were
\end{abstract}

in the PH domain and two were in the kinase domain of Btk. Three of these mutations were of the missense type, two of which altered the same codon in the PH domain; the third one was located in the kinase domain. The fourth mutation was a point deletion in the $\mathrm{PH}$ domain causing a frameshift followed by premature termination. The fifth mutation was a splice donor-site mutation within the kinase domain which could result in an exon skipping. In four of the five instances, mothers of the patients were shown to be obligate carriers. In one instance, a sibling sister was identified as a heterozygote establishing her as a carrier.

Conclusions: Functional consequences of the mutations causing frameshifts and altered splicing can be inferred directly. Functional consequences of the missense mutations were interpreted by 3-dimensional structural modeling of Btk domains. It is proposed that the two $\mathrm{PH}$ domain mutations will interfere with membrane localization while the kinase domain mutation will interfere with the enzymatic function of Btk. This study provides further insight into the role of Btk in XLA.

\section{INTRODUCTION}

$\mathrm{X}$-linked agammaglobulinemia (XLA) is the first immunodeficiency disorder to be described in humans (1). Symptoms are usually evident within the first year of life, although there are

Address correspondence and reprint requests to: Dr. Bratin K. Saha, Glenn Building/GHS, 69 Butler Street, SE, Atlanta, GA 30303, USA. Tel: 404-616-7424; Fax: 404-6167455; e-mail: bsaha@emory.edu instances of late onset of the disease (2). Affected individuals (males) encounter recurrent bacterial infections as they are unable to mount an antibody response due to a lack of serum immunoglobulin (Ig) of all classes. Unless treated aggressively with antibiotics and intravenous immunoglobulin (IVIG), the disease is often fatal. At the cellular level, XLA is associated with a severe deficit of circulating B cells (and an absence of plasma cells), 
although the level of pro-B cells in the bone marrow is normal, indicating a disruption in the pathway of B cell development.

Analysis of B cells from asymptomatic female carriers (heterozygotes) shows a nonrandom pattern of $\mathrm{X}$ chromosome inactivation $(3$, and references therein). The $\mathrm{T}$ cells and cells of other lineages display the expected pattern of random $\mathrm{X}$-inactivation, implying that the defect in XLA is intrinsic to B cells. Thus the gene affected in XLA must be critical for the maturation and differentiation of pro-B cells to B cells. Recently, Tsukada et al. (4) and Vetrie et al. (5) demonstrated that the typical XLA phenotype is due to mutations in an intracellular protein tyrosine kinase gene named Btk (Bruton's tyrosine kinase). The same gene is also mutated in a similar, albeit milder, disease in mice known as Xid (X-linked immunodeficiency; refs. 6,7). Xid mice also exhibit the skewed X-inactivation in their B cells, but not in $\mathrm{T}$ cells, as in XLA (8).

Btk belongs to the Tec family of cytosolic protein tyrosine kinases. Members of this family are characterized by an $\mathrm{N}$-terminal sequence consisting of a pleckstrin homology $(\mathrm{PH})$ domain and a Tec homology (TH) domain, followed by the Src homology domains $\mathrm{SH} 3$ and $\mathrm{SH} 2$, and finally, the C-terminal catalytic kinase domain. The human Btk gene consists of 19 exons and spans over $37 \mathrm{~kb}$ of DNA (9-12). The cDNA encodes 659 amino acids.

A database (BTKbase) for XLA mutations has been established recently by an international study group that can be accessed freely on the Internet (13; and references therein). The version 4 lists a staggering 228 unique mutations in 368 patients from 318 unrelated families. Thus most XLA mutations have evolved independently and many are new. Approximately onethird of the mutations are of the missense type and the rest consist of insertions, deletions, and nonsense and splice-site mutations. The distribution of mutations is fairly uniform throughout the length of the coding sequence so that all the functional domains are affected almost equally. Thus the kinase domain comprising $40 \%$ of the length accounts for approximately $45 \%$ of the mutations. A recent three-dimensional modeling study of the Btk kinase domain with eight point mutations, between residues 430 and 613 , revealed that all these mutations had clustered together on one face of the kinase domain (14), explaining the functional basis of these mutations. Structural consequences of several other mutations encompassing different functional do- mains of Btk have since been reported (for a review see ref. 15). In this paper we describe five novel mutations and predict their structural consequences. We also report three DNA polymorphisms, two of which are novel.

\section{PATIENTS, MATERIALS, AND METHODS}

\section{Patients}

All six patients had classical symptoms of XLA. They have been registered with BTKbase and are assigned unique patient identification numbers (PIN) and accession numbers. Patient P20 [PIN Y40N(1), Accession No. A0393] was first diagnosed with XLA at a somewhat late age of 8 years. There was no history of the disease in the family. Now at 21 years of age, he is receiving IVIG and is doing well. Patient P14 [Y40C(1), A0392] was diagnosed with XLA at around 2 years of age. His brother and a maternal uncle also had XLA. Patient P3 [D107X120(1), A0394] was diagnosed at the age of 3 years. His brother died of Pseudomonas sepsis at 16 months with autopsy findings suggestive of agammaglobulinemia. Now at 11 years of age, $\mathrm{P} 3$ is receiving IVIG and is doing well. Patient P21 [A508D(1), A0395] was diagnosed at the age of 5 years. There was no family history of XLA. Two patients, P11 [Intron 18(3a), A0396] and P12 [Intron 18(3b), A0397], are brothers. They were diagnosed around 1-2 years of age. Both are receiving IVIG and are doing well clinically.

\section{SSCP Analysis}

DNA was prepared from the whole blood of patients and their family members following a standard procedure. The putative promoter sequence and all 19 exons, including the respective exonintron junctions, of the Btk gene were amplified using primers described by Vorechovsky et al. (16). PCR was performed in $10 \mu \mathrm{l}$ of $1 \mathrm{X}$ reaction buffer (Fisher Scientific, Springfield, NJ) containing approximately $100 \mathrm{ng}$ DNA, $200 \mu \mathrm{M}$ each dNTP, $0.3 \mu \mathrm{M}$ each primer, $2.5 \mathrm{U}$ Taq DNA polymerase (Fisher Scientific) and 1-3 $\mu \mathrm{Ci} \alpha-\left[{ }^{32} \mathrm{P}\right]$ dCTP $(6000 \mathrm{Ci} / \mathrm{mmol})$. The reaction tubes were always loaded in the thermal cycler at $94^{\circ} \mathrm{C}$. The cycling started with a preheating step of $4 \mathrm{~min}$ at $94^{\circ} \mathrm{C}$ and concluded with an extension step of 5 min at $72^{\circ} \mathrm{C}$. The amplification was carried out over 30 cycles of $30 \mathrm{sec}$ at $94^{\circ} \mathrm{C}, 45 \mathrm{sec}$ at $57^{\circ} \mathrm{C}$, 
TABLE 1. Mutations in the Btk gene of XLA patients

\begin{tabular}{lllll}
\hline Patient & $\begin{array}{c}\text { Nucleotide } \\
\text { Change }^{a}\end{array}$ & $\begin{array}{c}\text { Amino Acid } \\
\text { Change }\end{array}$ & Exon/Intron/Domain & RFLP $^{b}$ \\
\hline P20 & T250A & Y40N & Exon 2/PH domain & None \\
P14 & A251G & Y40C & Exon 2/PH domain & None \\
P3 & del G451 & Frameshift & Exon 5/PH domain & None \\
P21 & C1655A & A508D & Exon 15/kinase domain & NcoI (-), BbsI (+) \\
P11, P12 & G2040 (+1)T & Skip exon 18 & Intron 18/kinase domain & $M n l$ (-), MseI (+) \\
\hline
\end{tabular}

${ }^{a}$ The nucleotide sequence designation is from Vetrie et al. (5).

${ }^{b}(-)$ indicates loss and $(+)$ indicates gain of the indicated restriction site due to the nucleotide change.

and $30 \mathrm{sec}$ at $72^{\circ} \mathrm{C}$. One microliter of the amplified DNA was mixed with $9 \mu$ l of the formamidedye loading solution and heated at $103^{\circ} \mathrm{C}$ for 3 min $(9,17)$. The single-stranded DNA was then resolved by PAGE ( $8 \%$ acrylamide/ $0.16 \%$ bisacrylamide containing $0,5 \%$ or $10 \%$ glycerol) in $0.5 \times$ TBE.

\section{DNA Sequencing}

The exons revealing conformational polymorphism were reamplified without any radiolabel. After resolving on an agarose gel, each ethidium bromide-stained band of interest was excised, and the DNA was eluted using a combination of Gel Nebulizer and Micropure filter (Amicon Inc., Beverly, MA). DNA sequencing was carried out in both directions using a fmol DNA sequencing system (Promega, Madison, WI) or a Sequitherm EXCEL DNA sequencing kit (Epicentre Technologies, Madison, WI) utilizing the same primers as in the amplification.

\section{Structural Analysis}

The structural consequences of the missense mutations were predicted based on the three-dimensional models of the $\mathrm{PH}$ and kinase domains of Btk described previously $(14,15,18)$. The models have been built based on X-ray crystal structures of related proteins, dynamin $\mathrm{PH}$ domain $(19,20)$ and cyclic AMP-dependent protein kinase $(21)$. The missense mutations were introduced into the models and the outcome of the amino acid substitutions was predicted based on the knowledge about protein structure and function.

\section{RESULTS}

The six XLA patients (P20, P14, P3, P21, P1 1, and P12) from five unrelated families were analyzed. The patients P11 and P12 were sibling brothers. Mothers were analyzed in all instances except for P14. The sibling sister of P20 was also analyzed to assess her carrier status. In five cases the SSCP analysis revealed a conformational polymorphism only in one of the 19 exons. In one case, a conformational polymorphism was revealed in two different exons, one of which turned out to be a silent polymorphism. Two additional silent polymorphisms, including one in an intron, were also revealed. Tables 1 and 2 summarize the five mutations and the three polymorphisms, respectively, while Fig. 1 depicts the relative positions of all the mutations and polymorphisms in reference to the Btk functional domains.

\section{Mutations in the PH Domain}

Three of the six patients, P20, P14, and P3, displayed mutations in the $\mathrm{PH}$ domain. $\mathrm{P} 20$ and P14 showed missense substitutions in two adjacent nucleotides in exon 2 of the PH domain. In P20, a T-to-A transversion at nucleotide 250 (Fig. 2) would result in the amino acid substitution Y40N. The mother and a sister of P20 were found to be heterozygous for T/A at nucleotide 250 (Fig. 2), indicating that they both were carriers and confirming the mutation in the proband. P14 had an A-to-G transition at nucleotide 251 (Fig. 2) substituting amino acid Y40C. Thus both mutations affected the same residue at codon 40 , although substituting different amino acids. No female family members were available for P14. 
TABLE 2. Polymorphisms in the $B t k$ gene

\begin{tabular}{clll}
\hline Polymorphism & Nucleotide Position $^{\boldsymbol{a}}$ & Exon/Intron/Domain & RFLP (+/-) $^{\boldsymbol{b}}$ \\
\hline $\mathrm{C} / \mathrm{T}$ & $273(+11)$ & Intron 2/PH domain & TaqI $(+/-)$ \\
$\mathrm{C} / \mathrm{T}$ & 1086 & Exon 11/SH2 domain & AlwNI $(-/+)$ \\
$\mathrm{C} / \mathrm{T}$ & 2031 & Exon 18/kinase domain & AlwNI $(-/+)$ \\
& & & \\
\hline
\end{tabular}

${ }^{a}$ The nucleotide sequence designation is from Vetrie et al. (5).

${ }^{b}( \pm / \pm)$ corresponds to $(\mathrm{C} / \mathrm{T})$ at the indicated location.

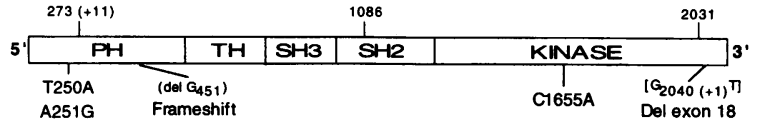

FIG. 1. Btk coding sequence showing approximate positions of the mutations (bottom) and polymorphisms (top)

The sequence starts at nucleotide 133 and ends at nucleotide 2109. The protein functional domains are indicated by $\mathrm{PH}$ (pleckstrin homology), TH (Tec homology), SH3 (Src homology 3), SH2 (Src homology 2 ), and kinase, respectively. Intronic alterations are noted at the nearest exonic positions.

An examination of exon 2 by SSCP in an additional 65 unrelated individuals $(105 \mathrm{X}$ chromosomes) revealed a mobility shift only in two instances resulting from an irrelevant silent polymorphism in intron 2 (see below). Thus the Y40N and the Y40C are unlikely to be some rare polymorphisms and are most likely to be the mutations causing XLA.

The mutation in $\mathrm{P} 3$ was also observed in the $\mathrm{PH}$ domain, but in exon 5. A point deletion of $\mathrm{G}$ at nucleotide 451 (data not shown) would lead to a frameshift introducing 13 missense amino acids after residue 106 before terminating prematurely. The truncated protein, if transcribed and translated, would lack most of the C-terminal sequences and is likely to be severely dysfunctional. The mother of P3 was heterozygous for the deletion allele (data not shown) confirming the mutation in the proband.

\section{Mutations in the Kinase Domain}

The other three patients (including two siblings) showed mutations in the kinase domain. P21 had a missense mutation in exon 15, a C-to-A transversion at nucleotide 1655 (data not shown), leading to the amino acid substitution A508D. In order to rule out that this was not a mere polymorphism, 65 individuals comprising both sexes ( $105 \mathrm{X}$ chromosomes) were screened for this mutation by SSCP analysis. None but P2 1 showed an altered mobility (data not shown), which implied that the base alteration in P21 is most likely a pathogenic mutation. The mother of P21 was a C/A heterozygote (data not shown), indicating her carrier status. The C-to-A nucleotide substitution results in the loss of $N c o$ I and StyI restriction sites as well as the gain of $B b s I$ and MboII restriction sites providing tools for rapid screening and confirmation of this mutation.

The second mutation in the kinase domain was revealed by the two siblings, P11 and P12. The G-to-T transversion at the first nucleotide of intron 18 at position $2040(+1)$ (data not shown) would result in the loss of an $M n l$ r restriction site and gain of an $\mathrm{MseI}$ restriction site. The mother of P1 1 and P12, as can be expected, was a carrier (data not shown). This splice donor-site mutation is expected to cause an aberrant splicing that would result in skipping of exon 18 followed by a frameshift (Fig. 3, ref. 22). This would lead to premature termination after the insertion of three missense amino acids following residue 583. The resulting truncated protein would lack several amino acids from the C-terminus of Btk and is likely to be functionally inactive as deletion of a few terminal residues can lead to XLA (16). This mutation could also lead to novel splicing and unusual transcripts (23).

\section{DNA Polymorphisms}

During the course of this work we encountered three $\mathrm{C} / \mathrm{T}$ silent polymorphisms that were initially detected as SSCPs and subsequently confirmed by DNA sequencing (Table 2). The first of 

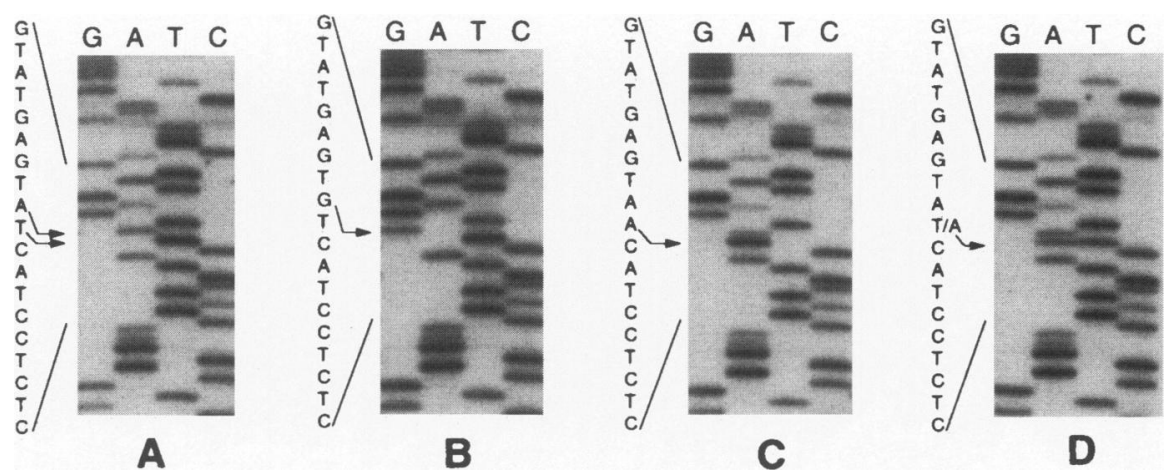

FIG. 2. Partial DNA sequence of Btk exon 2 showing mutations in P14 and P20, and heterozygosity in carriers from the family of $P 20$

(A) normal sequence; (B) A251G in P14; (C) T250A in P20, and (D) T/A heterozygosity at position 250 in the mother/sister of P20. The sequence is read from nt 241 (bottom) to nt 259 (top). Nucleotides T250 and A251 are indicated by arrows in the normal subject (A); the corresponding alterations are marked in the patients and carriers $(B-D)$.

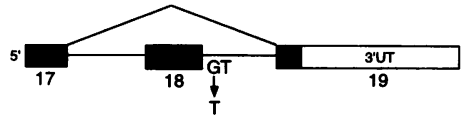

FIG. 3. Altered splicing of the Btk transcript in P11 and P12

Exons 17,18 , and 19 are shown as rectangles. Introns are shown as lines (not in scale). The donor splice-site mutation, $\mathrm{G}$ to $\mathrm{T}$, in intron $18(+1)$ is predicted to cause splicing of exon 17 with exon 19 , skipping exon 18 altogether.

these polymorphisms, at nucleotide $273(+11)$ in intron 2 (data not shown), would lead to a TaqI RFLP. The second polymorphism at nucleotide 1086 in exon 11 of the SH2 domain and the third polymorphism at nucleotide 2031 in exon 18 of the kinase domain (data not shown) would each result in an AlwNI RFLP. Although the polymorphism at nucleotide 2031 has been observed on several occasions $(16,24,25)$, the polymorphisms at nucleotides 1086 and $273(+11)$ appear to be novel. When we measured the frequency of the $\mathrm{C}$ and $\mathrm{T}$ nucleotides at the $273(+11)$ locus, 63 of 65 individuals ( $105 \mathrm{X}$ chromosomes) showed a $\mathrm{C}$ at this location, indicating that the $\mathrm{T}$ allele is extremely rare. The polymorphism at nucleotide 1086 was not studied further. Polymorphisms at nucleotides $273(+11)$ and 1086 both involved the $\mathrm{CpG}$ dinucleotide, known to be a mutational hotspot.

In heterozygotes these polymorphisms provide additional genetic markers for tracking the disease and wild-type alleles. For example, with the P1l family (the two affected siblings and their mother), one can simultaneously follow the polymorphic nucleotide at 2031 and the adjacent mutation at nucleotide $2040(+1)$, as both can be displayed on the same sequencing gel. Indeed, the mother was heterozygous for $\mathrm{C} / \mathrm{T}$ whereas P11 and, expectedly, P12 showed a C at the corresponding position, thus identifying the latter as the disease allele. Polymorphisms also help safeguard against inadvertent mix-up of DNA samples, as a polymorphism detected in an individual can be regarded as a signature for the entire family. Polymorphisms associated with RFLPs (Table 2) can be identified directly by restriction digestion, following PCR-amplification or followed by Southern blot hybridization, and are extremely useful for linkage mapping in the absence of a known mutation (26). Finally, an exonic polymorphism (Table 2 ) can be conveniently utilized as an EST to track RNA transcripts and to monitor $\mathrm{X}$ chromosome inactivation in different cell/tissue types in a heterozygote (8).

\section{DISCUSSION}

Although over 200 unique mutations in XLA patients have been listed in the BTKbase (13), the five mutations along with two of the polymorphisms reported here are novel. The functional consequence of the mutation in P3 or P11/P12, causing a frameshift or altered splicing followed by premature termination, is predictable. For example, the level of the altered mRNAs or the corresponding proteins could be severely abated because of an accelerated rate of their degradation resulting from intracellular surveillance (27). Alternatively, the truncated 


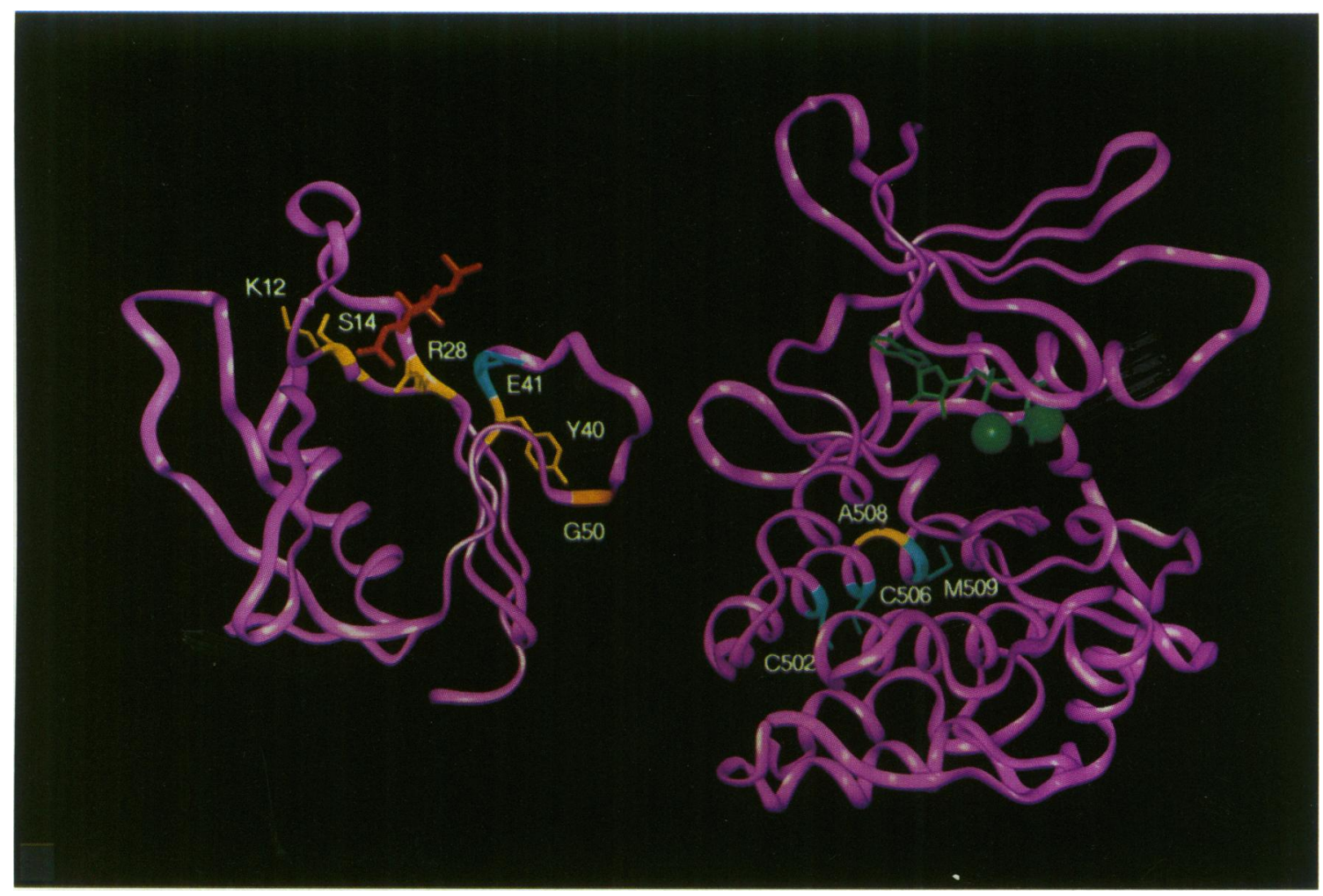

FIG. 4. Mutations in the Btk domains

Ribbons are running along the backbone of the domains. Left: the PH domain. Residues Y40, E41, and G50 are shown in yellow, cyan, and orange, respectively. The side chains of residues K12, S14 and R28, mutated in XLA and involved in phosphatidyl inositol (indicated in red) binding, are also highlighted in yellow. Right: the kinase domain. ATP and Mg2+ ions are shown in green and A508 is shown in yellow. Three other residues, C502, C506, and M509 in $\alpha$ helix-E, also mutated in XLA, are indicated in cyan. Modeling of the domains has been published previously $(14,18)$.

proteins could either be nonfunctional or function sparingly, depending upon the degree of truncation and the concomitant change in conformation. In contrast, functional consequences of the three missense mutations, two in the $\mathrm{PH}$ domain (P20 and P14) and one in the kinase domain (P21), are not immediately apparent (Table 1 and Fig. 1). Recently, the $\mathrm{PH}$ and kinase domains from a variety of proteins, including Btk, have been studied by X-ray crystallography, NMR spectroscopy, and molecular modeling. We have utilized the 3-D models to interpret the functional consequences of the missense mutations.

Discovered only recently the $\mathrm{PH}$ domain has been identified in over 100 proteins (for a review see ref. 28). It is intriguing that Tec family members are the only protein tyrosine kinases to contain a $\mathrm{PH}$ domain and Btk is the only protein known where mutations in the $\mathrm{PH}$ domain have a phenotype. The function of the $\mathrm{PH}$ domains has been difficult to study because these modules of approximately 100 amino acids have a diverse sequence. Only one residue, W124 in Btk, is almost invariant in all the $\mathrm{PH}$ domains. The $\mathrm{N}$ terminal half of several $\mathrm{PH}$ domains has been shown to bind phosphoinositides and may be responsible for membrane localization (29-34). The binding residues have been mapped in $\mathrm{PH}$ domains of pleckstrin, spectrin, dynamin, and Btk $(32,33,35,36)$. Using a biosensor-based assay, Salim and colleagues (34) observed that the Btk PH domain specifically bound liposomes containing phosphatidylinositol-3,4,5-triphosphate (IP3) and that the binding was abolished by the mutation R28Y. Fukuda and colleagues (30) demonstrated that the Btk PH domain can bind to inositol 1,3,4,5-tetrakisphosphate (IP4) and that the binding is dramatically reduced by the XLA mutations F25S, R28H, T33P, V64F, V113D, as well as $\mathrm{R} 28 \mathrm{C}$, originally discovered in Xid $(6,7,13)$. Interestingly, the constitutively acti- 
vated transforming mutation $\mathrm{E} 4 \mathrm{~K}$, produced by in vitro mutagenesis (37), binds IP4 (and IP5) with the same affinity, and IP6 with a 2-fold higher affinity than the wild type Btk. The fate of the Y40N and Y40C mutations has not been determined yet; however, these alterations are likely to result in a significantly low affinity for IP4 as evidenced by their loss-of-function phenotype in XLA. The fact that both mutations occur at the same residue implies that $\mathrm{Y} 40$ is critical for normal function of the $\mathrm{PH}$ domain.

Modeling of the 3-D structure of Btk and clustering of XLA-causing mutations suggested the PH domain to have a binding site (18) which, according to the recent X-ray structure, would bind phosphoinositides (32). Phosphatidylinositol-1,4,5 triphosphate (IP3) has been modeled into the Btk PH domain based on the binding site in PLC- $\delta_{1}(15,38)$. Residues K12, S14, and R28, involved in XLA mutations and residue E41 involved in the gain-of-function mutation, all appear to be involved in PLC- $\delta_{1}$ type phosphoinositide binding. In the structural model of the $\mathrm{PH}$ domain, these residues, along with Y40, cluster on the same plane of the binding site (Fig. 4, left). The side chain of $\mathrm{Y} 40$ is buried by the loop between $\beta$-strands 3 and 4 , and the hydroxyl group of $\mathrm{Y} 40$ could potentially form a hydrogen bond with the backbone of G50 (Fig. 4, left). Mutations at this site will presumably have structural consequences on the conformation of the loop and the phosphatidylinositol binding site and maybe of the whole domain, and they are likely to be deleterious for the normal function of Btk.

Unlike the PH domains, the kinase domains in both serine/threonine and tyrosine kinases share several conserved hallmark residues (39). The molecular structure of several kinases from both families has been determined by a variety of means. The three-dimensional scaffolding of these proteins is similar despite very low overall sequence similarities. The kinase domain consists of two lobes. The $\mathrm{N}$-terminal smaller upper lobe comprising mainly the five-stranded antiparallel $\beta$-sheet is primarily responsible for ATP binding. The C-terminal larger lower lobe comprising mostly seven $\alpha$-helices is a major substrate-binding region. The ATP, donating the phosphate group, is bound between the lobes. The regulation of protein kinase activities is a multistep process in which translocation of the upper lobe plays a major role. In the inactive form, the upper part is twisted relative to the lower lobe. In the active form, the ATP and essential $\mathrm{Mg}^{2+}$ ions are in their positions between the lobes.

Although XLA-causing kinase domain mutations are scattered along the sequence, they are primarily on one face of the domain, in the side where ATP and substrate are bound (14). Most of the mutations presumably have structural consequences $(13-15,38,40)$. The residue 508 is located in $\alpha$-helix $\mathrm{E}$ in the lower lobe of the kinase domain (Fig. 4, right). Three other mutations involving residues C502, C506, and M509 are also located in this helix (Fig. 4, right; and refs. $9,16,25,38,41-43)$. Replacement of any of these residues, which are at corresponding positions in successive turns, causes a structural alteration. The mutation A508D introduces a charged residue in the hydrophobic core of the domain and, therefore, is likely to alter its conformation. Polar residues are not allowed to reside in a protein core unless the charge can be neutralized. Another, compensatory mutation would be required to tolerate the A508D mutation.

\section{ACKNOWLEDGMENTS}

We are grateful to Dr. Michael Hebert for critical reading of the manuscript. This work was supported by Emory Medical Care Foundation (B.K.S.), and Biocentrum Helsinki and Finnish Academy (M.V.).

\section{REFERENCES}

1. Bruton OC (1952) Agammaglobulinemia. Pediatrics 9: 722-728.

2. Kornfeld SJ, Haire RN, Strong SJ, et al. (1996) A novel mutation (Cys ${ }^{145}$ to stop) in Bruton's tyrosine kinase is associated with newly diagnosed X-linked agammaglobulinemia in a 51-year-old male. Mol. Med. 2: 619-623.

3. Buckley RH (1994) Assessing inheritance of agammaglobulinemia. New Engl. J. Med. 330: 1526-1528.

4. Tsukada S, Saffran DC, Rawlings DJ, et al. (1993) Deficient expression of a B cell cytoplasmic tyrosine kinase in human X-linked agammaglobulinemia. Cell 72: 279-290.

5. Vetrie D, Vorechovsky I, Sideras $\mathrm{P}$, et al. (1993) The gene involved in X-linked agammaglobulinaemia is a member of the SRC family of protein-tyrosine kinases. Nature (Lond.) 361: 226-233. 
6. Rawlings DJ, Saffran DC, Tsukada S, et al. (1993) Mutation of unique region of Bruton's tyrosine kinase in immunodeficient XID mice. Science 261: 358-361.

7. Thomas JD, Paschalis S, Smith CIE, Vorechovsky I, Chapman V, William PE (1993) Colocalization of X-linked agammaglobulinemia and $X$-linked immunodeficiency genes. Science 261: 355-358.

8. Shanmugam V, Chapman VM, Sell KW, Saha BK (1996) A novel Tthlll I RFLP allows tracing of $\mathrm{X}$-chromosome inactivation in Xid heterozygote. Biochem. Genet. 34: 1729.

9. Hagemann TL, Chen Y, Rosen FS, Kwan S-P (1994) Genomic organization of the Btk gene and exon scanning for mutations in patients with X-linked agammaglobulinemia. Hum. Mol. Genet. 3: 1743-1749.

10. Ohta Y, Haire RN, Litman RT, et al. (1994) Genomic organization and structure of Bruton's agammaglobulinemia tyrosine kinase: Localization of mutations associated with varied clinical presentations and course in X-chromosome-linked agammaglobulinemia. Proc. Natl. Acad. Sci. U.S.A. 91: 9062-9066.

11. Rohrer J, Parolino O, Belmont JW, Conley ME (1994) The genomic structure of human $B t k$, the defective gene in X-linked agammaglobulinemia. Immunogenetics 40: 319-324.

12. Sideras P, Muller S, Shiels H, et al. (1994) Genomic organization of mouse and human Bruton's agammaglobulinemia tyrosine kinase (Btk) loci. J. Immunol. 153: 5607-5617.

13. Vihinen M, Belohradsky BH, Haire RN, et al. (1997) BTKbase, mutation database for Xlinked agammaglobulinemia (XLA). Nucl. Acids Res. 25: 166-171.

14. Vihinen M, Vetrie D, Maniar HS, et al. (1994) Structural basis for chromosome X-linked agammaglobulinemia: A tyrosine kinase disease. Proc. Natl. Acad. Sci. U.S.A. 91: 1280312807.

15. Vihinen M, Mattsson P, Smith CIE (1997) Btk, the tyrosine kinase affected in X-linked agammaglobulinemia. Frontiers Biosci. 2: d384-d399.

16. Vorechovsky I, Luo L, de Saint Basile G, Hammarstrom L, Webster ADB, Smith CIE (1995) Improved oligonucleotide primer set for molecular diagnosis of X-linked agammaglobulinemia: Predominance of amino acid substitutions in the catalytic domain of Bruton's tyrosine kinase. Hum. Mol. Genet. 4: 2403-2405.

17. Conley ME, Fitch-Hilgenberg ME, Cleveland
JL, Parolini O, Rohrer J (1994) Screening of genomic DNA to identify mutations in the gene for Bruton's tyrosine kinase. Hum. Mol. Genet. 3: 1751-1756.

18. Vihinen M, Zvelebil MJJM, Zhu Q, et al. (1995) Structural basis for pleckstrin homology domain mutations in X-linked agammaglobulinemia. Biochemistry 34: 1475-1481.

19. Downing AK, Driscoll PC, Gout I, Salim K, Zvelebil MJ, Waterfield MD (1994) Threedimensional structure of the pleckstrin homology domain from dynamin. Curr. Biol. 4: 884-891.

20. Ferguson KM, Lemmon MA, Schlessinger J, Sigler PB (1994) Crystal structure at $2.2 \mathrm{~A}$ resolution of the pleckstrin homology domain from human dynamin. Cell 79: 199209.

21. Knighton DR, Zheng J, Eyck LFT, et al. (1991) Crystal structure of the catalytic subunit of cyclic adenosine monophosphate-dependent protein kinase. Science 253: 407-414.

22. Jin H, Webster ADB, Vihinen M, et al. (1995) Identification of Btk mutations in 20 unrelated patients with $\mathrm{X}$-linked agammaglobulinemia (XLA). Hum. Mol. Genet. 4: 693-700.

23. Haire RN, Ohta Y, Strong S, et al. (1997) Unusual patterns of exon skipping in Bruton tyrosine kinase are associated with mutation involving the intron $173^{\prime}$ splice site. Am. J. Hum. Genet. 60: 798-807.

24. Bradley LAD, Sweatman AK, Lovering RC, et al. (1994) Mutation detection in the Xlinked agammaglobulinemia gene, Btk, using single-strand conformation polymorphism analysis. Hum. Mol. Genet. 3: 79-83.

25. de Weers M, Mensink RGJ, Kraakman MEM, Schurman RKB, Hendriks RW (1994) Mutation analysis of the Bruton's tyrosine kinase gene in X-linked agammaglobulinemia: Identification of a mutation which affects the same codon as is altered in immunodeficient Xid mice. Hum. Mol. Genet. 3: 161-166.

26. Kwan S-P, Walker AP, Hagemann T, Gupta S, Vayuvegula B, Ochs HD (1994) A new RFLP marker, SP282, at the Btk locus for genetic analysis in X-linked agammaglobulinemia families. Prenat. Diagn. 14: 493-496.

27. Maquat LE (1996) Defects in RNA splicing and the consequence of shortened translational reading frame. Am. J. Hum. Genet. 59: 279-286.

28. Shaw G (1996) The pleckstrin homology do- 
main: An intriguing multifunctional protein module. BioEssays 18: 35-46.

29. Ferguson KM, Lemmon MA, Schlessinger J, Sigler PB (1995) Structure of the high affinity complex of inositol triphosphate with a phospholipase C pleckstrin homology domain. Cell 83: 1037-1046.

30. Fukuda M, Kojima T, Kabayama H, Mikoshiba K (1996) Mutation of the pleckstrin homology domain of Bruton's tyrosine kinase in immunodeficiency impaired inositol 1,3,4,5-tetrakisphosphate binding capacity. J. Biol. Chem. 271: 30303-30306.

31. Harlan JE, Hajduk PJ, Yoon HS, Fesik SW (1994) Pleckstrin homology domains bind to phosphatidyl inositol-4,5-bisphosphate. $\mathrm{Na}$ ture 371: 168-170.

32. Hyvönen $M$, Macias MJ, Nilges $M$, Oschkinat H, Saraste M, Wilmanns M (1995) Structure of the binding site for inositol phosphates in a PH domain. EMBO J. 14: 4676-4685.

33. Hyvönen M, Saraste M (1997) Structure of $\mathrm{PH}$ domain and Btk motif from Bruton's tyrosine kinase: Molecular explanation for $\mathrm{X}$ linked agammaglobulinemia. $E M B O$ J. 16: 3396-3404.

34. Salim K, Bottomly MJ, Querfurth E, et al. (1996) Distinct specificity in the recognition of phosphoinositides by the pleckstrin homology domains of dynamin and Bruton's tyrosine kinase. EMBO J. 15: 6241-6250.

35. Harlan JE, Yoon HS, Hajduk PJ, Fesik SW (1995) Structural characterization of the interaction between a pleckstrin homology domain and phosphatidylinositol-4,5-bisphosphate. Biochemistry 34: 9859-9864.

36. Zheng J, Cahill SM, Lemmon MA, Fushman

Communicated by J. Leiden. Accepted May 8, 1997.
D, Schlessinger J, Cowburn D (1996) Identification of the binding site for acidic phospholipids on the $\mathrm{PH}$ domain of dynamin: Implications for stimulation of GTPase activity. J. Mol. Biol. 255: 14-21.

37. Li T, Tsukada S, Satterthwaite A, et al. (1995) Activation of Bruton's tyrosine kinase (Btk) by a point mutation in its pleckstrin homology $(\mathrm{PH})$ domain. Immunity 2: 451-460.

38. Mattsson PT, Vihinen M, Smith CIE (1996) $\mathrm{X}$-linked agammaglobulinemia (XLA): A genetic tyrosine kinase (Btk) disease. BioEssaays 18: $825-834$.

39. Hanks SK, Hunter T (1995) The eukaryotic protein kinase superfamily: Kinase (catalytic) domain structure and classification. FASEB J. 9: 576-596.

40. Vihinen M, Smith CIE (1996) Structural aspects of signal transduction in B cells. Crit. Rev. Immunol. 16: 251-274.

41. Conley ME, Rohrer J (1995) The spectrum of mutations in Btk that cause X-linked agammaglobulinemia. Clin. Immunol. Immunopathol. 76: S1192-S197.

42. Hasimoto S, Tsukada S, Matsushita, M, et al. (1996) Identification of Bruton's tyrosine kinase (Btk) gene mutations and characterization of the derived proteins in $35 \mathrm{X}$-linked agammaglobulinemia families: A nationwide study of Btk deficiency in Japan. Blood 88: 561-573.

43. Vorechovsky I, Luo L, Hertz JM, et al. (1997) Mutation pattern in the Bruton's tyrosine kinase gene in 26 unrelated patients with $\mathrm{X}$-linked agammaglobulinemia. Hum. Mutat. 9: 418-425. 